\title{
Prevention and management of malaria in under-five children in Tanzania: a review
}

\section{TELEMU KASSILE}

Department of Economics, College of Arts and Social Sciences, University of Dar es Salaam, P. O. Box 35045, Dar es Salaam, Tanzania

E-mail: telemuk@yahoo.com

\begin{abstract}
In 2010, an estimated 216 million cases and 655,000 deaths due to malaria occurred worldwide. Of these, about $81 \%$ and $91 \%$ respectively were reported from the African Region, mostly affecting children under-the age of five. In Tanzania, malaria remains to be the leading cause of high morbidity and mortality. It is responsible for more than one-third of all deaths, $39.4 \%$ of all health care visits, and $33.4 \%$ of all hospital admissions among children under the age of 5 years. The objective of this article was to provide a systematic review of the literature to summarize the knowledge of the most important aspects of prevention and management of malaria in under-five children in Tanzania. The focus was on health care seeking decision-making and understanding the magnitude of the gap between ownership and use of insecticide treated mosquito nets (ITNs). The review considered published and unpublished literature covering a period from 2002 to the end of July 2012. The information used in this review was obtained through a Google search using specific keywords. Results show that across zones the difference between ownership and use of ITNs among under-fives was insignificant. This finding is consistent with a reduction in the proportion of children with suspected of malaria taking antimalarial drugs. Nevertheless, differences exist between zones in terms of both coverage of ownership and use of ITNs. Regarding health care seeking-decision making the literature reviewed indicated that caretakers generally, had a good biomedical understanding of febrile illnesses both in terms of types and symptoms. However, a frequent belief was that symptoms related to convulsions were not associated with malaria, hence cannot be cured in modern health care system. Moreover, some caretakers self-treated symptoms, which trigger others to seek medical care, thus end up delaying to seek medical care. In additional, most of the studies reviewed were designed within the health belief framework. There is a dearth of evidence documenting adherence to prescribed medications.
\end{abstract}

Keywords: malaria, prevention, management, under-five children, Tanzania

\section{Introduction}

Malaria is still a major global health problem and one of the leading causes of mortality and morbidity in Sub-Saharan Africa. In 2010, an estimated 216 million cases and 655,000 deaths of malaria occurred worldwide, of which about $81 \%$ and $91 \%$ respectively were in the African Region. Most of the cases and deaths were reported in $<5$ year children (WHO, 2011). In Tanzania, malaria continues to be the leading cause of high morbidity and mortality (Rugemalila et al., 2006). The disease is responsible for more than one-third of all deaths, $39.4 \%$ of all health care visits, and $33.4 \%$ of all hospitalizations among children under the age of five (Mboera et al., 2007).

In an attempt to address the huge global malaria problem, the World Health Organization launched Roll Back Malaria (RBM) programme in 1998. In recent years, funds from the Global Fund to Fight AIDS, Tuberculosis and Malaria, the World Bank, the US President's Malaria Initiative, and bilateral donors have become available on a scale exceeding that of the first 
efforts to eradicate malaria in the 1950s and 1960s (O'Meara et al., 2010). One area, which has received particular global financial resources in recent years, is concerned with achieving high coverage of core malaria control interventions in malaria-endemic countries. Insecticide-treated mosquito nets (ITNs), indoor-residual spraying (IRS), Artemisinin-based combination therapy (ACT), and intermittent preventive treatment of malaria during pregnancy (IPTp) are the recommended interventions for prevention and management of malaria (WHO, 2008). ITNs and IRS prevent malaria infection by reducing the possibility of an individual to be bitten by a malaria-carrying mosquito (Eisele et al., 2010). ACT has the distinct benefit of reducing malaria transmission by decreasing gametocyte carriage while curing the disease (Barnes et al., 2009). Beginning 2005 through 2009, numerous countries made a significant progress in scaling-up of prevention intervention programmes (Steketee \& Campbell, 2010).

In Tanzania, in response to global commitments to reduce the burden of malaria, the National Malaria Medium Term Strategic Plan (2008-2013) puts emphasis on five strategic approaches namely: (i) malaria diagnosis and treatment; (ii) malaria prevention supportive strategies; (iii) Surveillance, Monitoring and Evaluation; (iv) Behavioural Change Communication and Community-based malaria control; and (v) Regional/District support and capacity building (MoHSW, 2009). In line with the health sector and local government reform, scaling-up of malaria control interventions has been one of the priority areas in recent years. For instance, in order to rapidly scale-up ownership and use of ITNs across communities especially in the lowest income categories, several strategies, including social marketing of ITNs, voucher scheme (targeting mainly pregnant women and infants), and a free net distribution campaign for under-five children, were adopted (TSPA, 2007).

Unlike many childhood illnesses, malaria can progress from mild to severe illness within few hours of the onset of the symptoms (Munthali, 2005). In this respect, recognition of danger signs and timely action in seeking medical care is essential to effective disease management and achievement of a significant reduction in severe morbidity and likelihood of death (Teerawichitchainan \& Phillips, 2008). However, health care seeking decision-making entails a sequence of stages (Mwabu, 1986; Suchman, 1965; Hill et al., 2003; Pokhrel \& Sauerborn, 2004; Pokhrel et al., 2005). The sequence of stages begins with recognition of symptoms thereafter formulation of treatment strategy (Salah et al., 2007). One aspect of significant importance to health care services utilization especially among children, unlike adults is that for the former subgroup, all the decision-making stages depend mostly on their caretakers (Akin et al., 1981). Wiseman et al. (2008) noted that a considerable amount of malariarelated deaths among under-five children in SSA occur because of delays in seeking medical care and adherence to prescribed medications. In the context of Tanzania, mothers in Dar es Salaam waited for at least 48 hours after onset of fever before they took their children to a health facility (Kamat \& Nyato, 2010). Health care seeking behaviour and socio-economic status, which determines access to health care services, are among the factors, which affect the utilization of health care services in developing countries around the world (Getahun et al., 2010).

Maslove et al. (2009) examined the literature on barriers to the effective prevention and management of malaria in Africa and found that insufficient understanding of the cause and transmission of malaria, the belief that malaria cannot be prevented, and the use of inefficient prevention practices were the most reported barriers to the effective prevention of malaria 
especially in children. The authors hence concluded that large-scale malaria control programmes must take into account the local social and contextual factors in which the intervention programmes are being implemented. The objective of this article was to provide a systematic review of the literature to summarize the knowledge of the most important aspects of prevention and management of malaria in under-five children in Mainland Tanzania focusing on health care seeking decision-making and the gap between ownership and use of ITNs among under-five children across geographic zones.

\section{Materials and Methods}

The review considered published and unpublished literature. However, the review was restricted to studies covering a period from 2002 to end of July 2012 and which included a component on prevention or treatment of malaria in under-five children. The review period primarily overlays closely to the decade (2001-2010) of rolling back malaria in developing countries and the Tanzania National Malaria Medium Term Strategic Plans of 2002-2007 and 2008-2013. The focus was to understand the progress and magnitude of the gap between ownership of ITNs and use (disease prevention) among children less than five years of age and the percentage of under-five children getting timely treatment (disease management) across geographic areas in the country in relation to the RBM 2010 target. A Google search using keywords such as "prevention of malaria in under-five children in Tanzania", "management of malaria in under-five children in Tanzania, "prevention and management of malaria in under-five children in Tanzania", "health care services utilization in Tanzania", and "roll back malaria in Tanzania" were used to identify published literature pertaining to the subject. From within the identified published literature, the references were scrutinised to identify other articles of interest not included in the initial search through the keywords. Articles published before 2002 and after July, 2012 were not included in the review.

Technical reports were included in the review, restricting to reports from surveys, which were conceived to be comparable in terms of sample design. This restriction aimed at reducing variations in estimates that could be attributed to differences in methodology and timing of data collection between surveys. Moreover, those survey reports in which the sample design allowed estimation of particular malaria indicators such ownership and use of ITNs across geographic zones within Tanzania were included. Under this category, reports reviewed are (i) Tanzania Demographic and Health Survey 2004-05 (TDHS, 2005); (ii) Tanzania HIV/AIDS and Malaria Indicator Survey 2007-08 (THMIS, 2008); and (iii) Tanzania Demographic and Health Survey 2010 (TDHS, 2011). The reports were downloaded via http://www.measuredhs.com at appropriate stage during the course of writing of this manuscript. Information regarding proportion of households with at least one ITN and the under-five children sleeping under an ITN during the previous night was extracted. Information on timing of antimalarial drugs for children suspected of malaria was also extracted from the reports. The information was subsequently used to examine numerically and graphically changes (levels and trends) in ownership and use of ITNs as well as timing of antimalarial drugs among under-five children overall and across geographic zones. The country was classified into Western, North, Central, Southern Highlands, Lake Victoria, Eastern, and 
Southern. Each zone is formed by 2-4 regions, resulting into a total of 21 regions (at the time of the surveys). For easy of interpretation of information extracted from the reports and in order to discern patterns of features of interest, figures of survey year were plotted against percentage coverage, and zone against percentage coverage.

\section{Results}

\section{Levels and trends in ownership and use of ITNs}

The review revealed that the percentage of households with at least one ITN increased from $22.5 \%$ in the $2004-05$ survey to $38.3 \%$ in the $2007-08$ survey and to $63.4 \%$ in the 2010 survey. In terms of use, $15.9 \%, 24.8 \%$, and $63.9 \%$ of under-five children slept under an ITN the preceding night of the 2004-05, 2007-08, and 2010 surveys (Figure 1). Disaggregated into geographic zones, the percentages (both ownership and use) in the 2004-05 survey was generally lower than that of the 2007-08 and 2010 surveys, with the 2010 proportions overall being above those observed in the 2007-08 survey. In addition, the 2004-05 survey displayed a similar pattern to that of the 2007-08 survey. However, the magnitude of the gap between ownership of at least one ITN and use by under-five was smaller in the 2004-05 survey than in the 2007-08 survey.
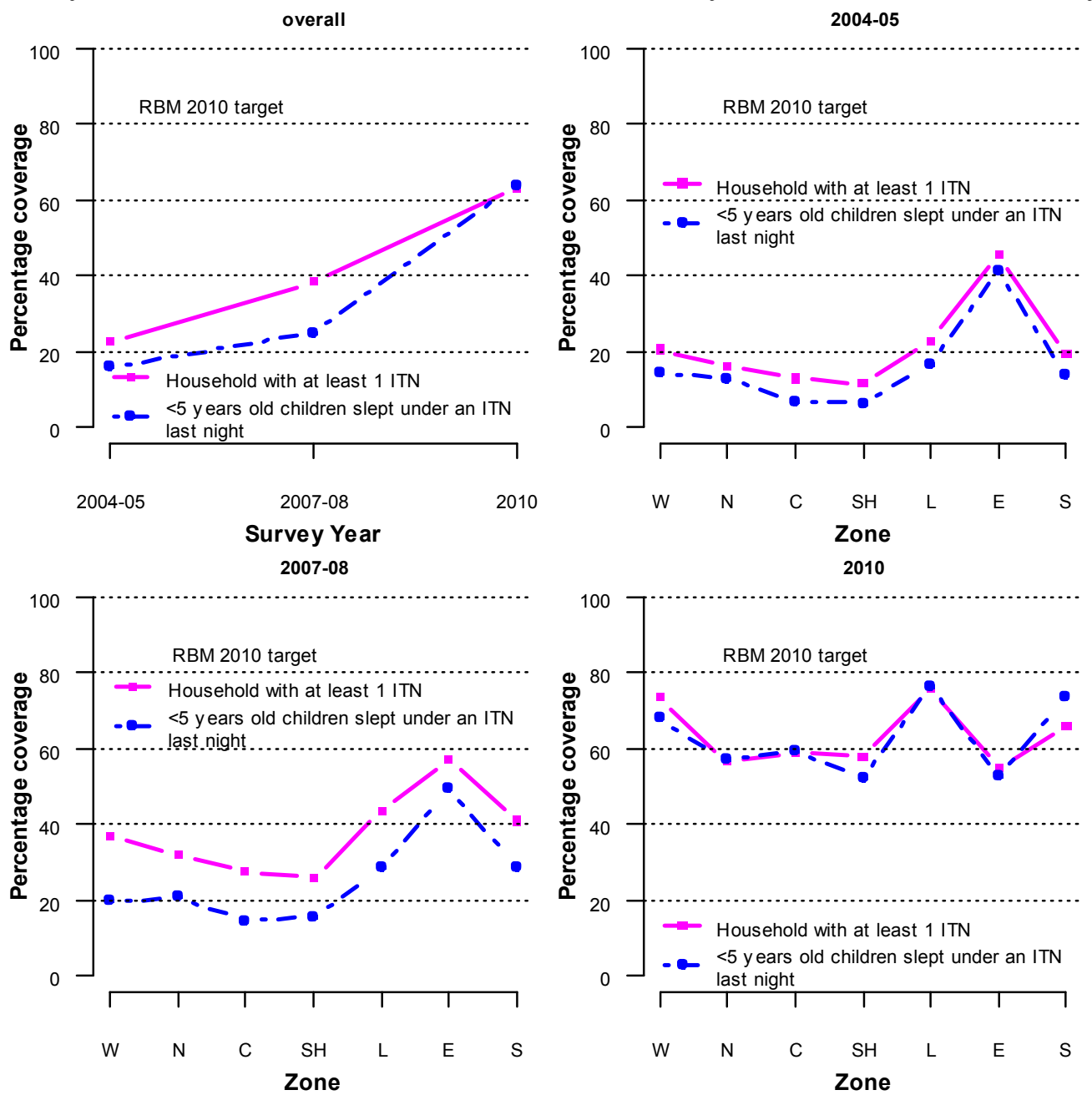

Figure 1: Levels and trends in ownership and use of ITNs in Mainland Tanzania, 2004-2010 
For the two surveys (2004-05 and 2007-08) and for both ownership and use of ITN, the eastern (E) zone, which includes the Dar es Salaam City, had the highest proportions compared to other zones. Furthermore, in recent years, as it is revealed in Figure 1, the magnitude of the gap between ownership and use of ITN was seen to disappear especially in the Northern $(\mathrm{N})$, Central (C), Lake Victoria (L), and Eastern zones. There was a marked increase in both ownership and use of ITN in the Lake Victoria zone from the values observed in the 2004-05 and 2007-08 surveys. Moreover, as seen in the plot for the 2010 survey (Figure 1), children in the Northern, Central, Southern Highlands, and Eastern zones were less likely to sleep under an ITN than in the Western, Lake and Southern zones. Overall, none of the seven zones reached the RBM 2010 target of $80 \%$ coverage.

\section{Caretakers' knowledge about malaria}

Caretakers had a good biomedical understanding of febrile illnesses both in terms of types and symptoms. In regard to types, caretakers made a clear distinction of three types of febrile illnesses namely, malaria ya kawaida ("uncomplicated malaria"), malaria kali ("severe malaria"), and degedege ("convulsions") (Makundi et al., 2006). In other places, uncomplicated malaria was commonly known as homa ya malaria (Comoro et al., 2003). In one study (Dillip et al., 2009) a febrile condition was identified as malaria, homa or degedege. Caretakers differentiated signs or symptoms of uncomplicated malaria from those of severe malaria in that the former was represented by a combination of ordinary fever, chills, body weakness, loss of appetite and vomiting while the later was characterized with high fever, shivering, loss of appetite, child becoming very weak and sleeping constantly. A mixture of high fever, loss of appetite, vomiting, rigid body and awkward movements of the limbs and eyes were mentioned as symptoms of convulsions. In contrast, in another study, convulsions, altered consciousness and coma were recognized as symptoms of severe malaria (Warsame et al., 2007). Moreover, caretakers associated vomiting and loss of appetite with malaria but perceived these symptoms as minor signs except when they appeared in combination with another more severe symptom (Warsame et al., 2007).

Perceived cause of mild and severe malaria and convulsions varied between caretakers. Some caretakers attributed mild and severe malaria to mosquitoes while others ascribed the signs or symptoms to change of weather, bad air and contaminated milk (Makundi et al., 2006). Symptoms of convulsions were frequently attributable to spiritual causes or supernatural forces (Comoro et al., 2003; Foster \& Vilendrer, 2009; Makundi et al., 2006; Warsame et al., 2007).

\section{Sources of care}

Caretakers sought care for episodes of malaria from different types of health care providers depending principally on their perceptions and beliefs regarding the illness (Savigny et al., 2004; Makundi et al., 2006; Warsame et al., 2007; Foster \& Vilendrer, 2009). For example, in a study conducted in Kilosa and Handeni, Makundi et al. (2006) found that caretakers who associated malaria to evil spirits sought care outside the modern health sector. In addition, other caretakers, upon recognition of symptoms, first responded to the illnesses by employing traditional care at home. Nsimba \& Rimoy (2005) found that about three-quarters of caretakers 
reported self-treating their sick children at home prior to visiting public health facilities. Nsimba et al. (2002) found that over half of children who were taken to health facilities for treatment were first self-treated at home mostly with antipyretics and chloroquine, one-fifth were taken to another health facility and only a few to traditional healers before they were brought to the health facilities. Eriksen et al. (2005) established that $8 \%$ of caretakers stocked antimalarials while only $4 \%$ reported self-treatment as the earliest course of action. Caretakers of children who experienced an episode of fever during the last 4 weeks sought care mostly (84\%) from public health facilities. The review observed a similar finding in rural villages in Kilosa District (Simba \& Kakoko, 2012). That is, febrile children received care largely from government health facilities. Other sources of care included ordinary shops, faith based organisations (FBO), home care, and accredited drug dispensing outlets (ADDO) (Simba \& Kakoko, 2012). Antimalarial drugs received were (in descending order of percentages) artemether-lumufantrine (ALu), sulphadoxine-pyrimethamine (SP), amodiaquine, quinine, and antipyretic (Simba \& Kakoko, 2012).

The review shows that, where necessary, traditional home management practices employed by caretakers included placing the child under bed, sponging or washing the child with cold water, and urinating on the ill child were among the practices adopted (Makundi et al, 2006). These traditional modus operandi were done with the goal of reducing the child's temperature. In some cases, paracetamol and aspirin were used for the same purpose as the traditional home remedies (Makundi et al., 2006). In situations of persisting symptoms, treatment outside home was then considered and where appropriate, the preferred treatment options were traditional healers or nearby health facility (Makundi et al., 2006). In southern Tanzania (Savigny et al., 2004), biomedical care, mostly from shops or government and nongovernment health facilities were reported as the most common source of treatment for suspected malaria cases. The choice of which provider to seek care from was independent of socio-demographic and economic characteristics of the household or presence or absence of convulsions.

\section{Timing of care}

As regards to timing of decision to seek treatment and use of antimalarials, the review has established that caretakers' subjective assessment of the illness was an important determining factor of timing of the decision to seek medical care at a health facility (Dillip et al., 2009). While well over two-thirds $(71 \%)$ of children with degedege received medical care within 24 hours after onset of first symptoms, only about $46 \%$ of children identified to have fever received timely use of health facility (Dillip et al., 2009). Moreover, approximately, 67\% of those with convulsions received antimalarial medications from the facility against $43 \%$ of those with fever. Knowledge of symptoms of convulsion and perceived causes were found to be less associated with both timing of health facility use and receipt of antimalarial from the facility. Instead, perceived quality of health care facilities and livelihood factors were the key determining factors of timely health facility use and antimalarials (Dillip et al., 2009). 


\section{Levels and trends in malaria treatment}

The proportion of under-five children suspected of malaria (with fever in the two weeks preceding the survey) who took antimalarial drugs has not changed much over the three surveys (Figure 2). In the 2004-05 survey, 58.2\% of those suspected of malaria took antimalarial drugs against $57.0 \%$, and $60.1 \%$ in the $2007-08$ and 2010 surveys, respectively. With respect to timing of antimalarial drugs following the onset of fever, the review noted that the percentages who took antimalarial drugs on the same or the following day indicates an overall declining trend. The numbers were 51.1\%, 34.4\%, and 41.4\% in the 2004-05, 2007-08, and 2010 surveys, respectively.
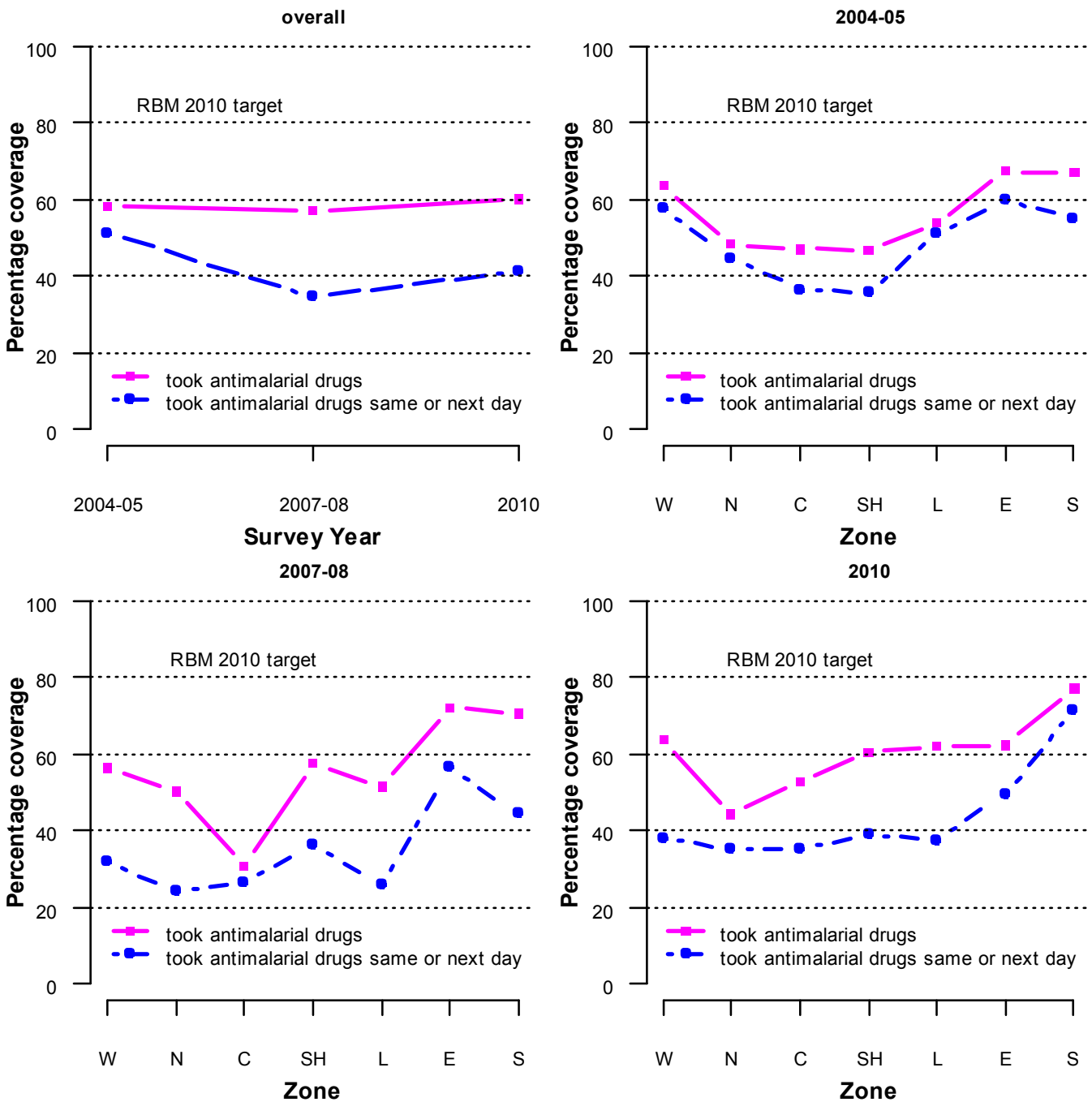

Figure 2: Levels and trends in malaria treatment in Mainland Tanzania, 2004-2010.

A comparison between zones within a survey indicated existence of some differences. In the 2004-05 survey, the Western and Eastern zones had the highest and almost equal (57.5 and 59.8) proportions of under-fives who took antimalarials on the same or next day after the onset of fever. The Lake and Southern zones had the next highest, also about equal percentages $(51.0 \%$ and $54.9 \%$ Southern), followed by the Northern zone $(44.6 \%)$. The lowest percentages were observed to be in the Central and Southern Highlands (36.5\% and 35.7\%). Examinations of the 
2007-08 and 2010 surveys also showed a similar pattern as that of the 2004-05 at least with regard to differences in percentage coverage between zones. However, differences were noticed between the 2004-05, 2007-08 and 2010 surveys. Relative to the observations in the 2004-05 survey, the percentage of under-five children who took antimalarial drugs on same or the next day in the 2007-08 survey was generally lower. A slight increase was noted in the 2010 survey but still on average, was relatively below the levels observed in the 2004-05. This pattern reflects the trend observed in the overall plot (Figure 1). The percentage coverage in the Southern and Eastern zones in 2010 was generally above that of the other zones. That is, $71.0 \%$ and $50.0 \%$ respectively, while those of the remaining zones were below the $40 \%$ line (Figure 2 ).

\section{Adherence to treatment}

The review identified two studies (Simba et al., 2010; Kachur et al., 2004) in which the concept of adherence to treatment advice was assessed. The review established that caretakers' decision whether to adhere to referral advice following pre-referral treatment was dependent on the health status of the child. Caretakers whose children did not improve were more likely to adhere to referral advice than those whose children showed marked improvement with prereferral treatment. Difficulties in reaching the health facilities, existence of other priorities, and concern about the quality of the health care facilities were among the reasons for non-adherence to referral advice for some caretakers of children whose children showed significant improvement with rectal artesunate. Lack of understanding of some caretakers on the need to proceed to health facilities was also a factor for failure of caretakers to adhere to referral advice after improvement with pre-referral treatment (Simba et al., 2010). Kachur et al. (2004) examined adherence to antimalarial combination therapy with sulfadoxine-pyrimethamine (SP) and artesunate (AS) in villages in the Rufiji District of Tanzania. The authors used self report, count of tablets, and a combination of self report and tablet count as measures of adherence. The found that, overall patients in the 24-hour arm were statistically significantly more likely to adhere to SP and AS than those in the 48-hour arm. The most common reason for non-adherence mostly among children was finishing all the tablets in less than 48 hours. Improvement of the patient's condition and skipping one dose also contributed to non-adherence.

\section{Discussion}

The present review revealed that the proportion of households owning at least an ITN and use among under-five children has increased over time-(from $22.5 \%$ in 2004-05 to $63.4 \%$ in 2010). However, the curve for use was generally below that for ownership, though the difference decreased with time and disappeared completely in 2010. This trend was equally true both in general and across zones over the three time points. The finding that the curve for ownership of ITNs was consistently above that of use among under-five children before the 2010 survey is similar to that reported elsewhere in Sub-Saharan Africa (Eng et al., 2010).

The rate of ownership and use of ITNs among under-five children across zones revealed some differences both in terms of ownership and use. Overall, for the three time points, the Northern, Central, and Southern Highlands zones had low rate of ITN ownership and use among under-five children. Mosquito net coverage and utilization has been linked to 
many factors, some of which overlap between settings. In Tanzania, factors such as availability of health care services (Mboera et al., 2006, 2008), and income, perception that insecticides have health effects to the users, and presence or absence of under-five children in the household (Matovu et al., 2009) have been observed. In Nigeria, Oresanya et al. (2008) found that the presence of health facility within the community, religion and wealth index by caregiver's education were among the predictors of use of ITN among under-five children. Availability of alternative malaria prevention products such as sprays, repellents, and coils as observed by McElroy et al. (2009) in the household could also impact the rate of utilization of ITNs.

Caretakers' biomedical knowledge of febrile illnesses was generally good. It was established that caretakers perceived a febrile condition in at least four different types namely: malaria, malaria ya kawaida (uncomplicated malaria), malaria kali (severe malaria), and degedege (convulsions) (Makundi et al., 2006). Malaria ya kawaida was also known as homa or homa ya malaria (Comoro et al., 2003). Caretakers were able to differentiate signs or symptoms of uncomplicated malaria from those of severe malaria as well as convulsions (Makundi et al., 2006). In all distinct types of malaria perceived by caretakers, fever was the most pronounced symptom. The only difference among the three types of febrile illnesses was that fever was subjectively perceived as "ordinary" (for uncomplicated malaria) and "high fever" for both severe malaria, and convulsions. In each perceived type, fever was considered with a combination of other symptoms. For example, ordinary fever with a combination of chills, body weakness, loss of appetite, body weakness, and vomiting was identified as mild malaria (Makundi et al., 2006). Symptoms like vomiting and loss of appetite were identified as malaria but treated as minor except when they appear together with a severe symptom (Warsame et al., 2007). However, it was unclear from the literature reviewed which symptoms were perceived as severe.

Frequently, caretakers characterized a febrile condition as degedege when there was a mixture of high fever, loss of appetite, vomiting, rigid body and awkward movements of the limbs and eyes (Makundi et al., 2006). However, a widespread belief among caretakers was that such signs were not connected with malaria but to supernatural forces (Comoro et al., 2003; Foster \& Vilendrer, 2009; Makundi et al., 2006; Warsame et al., 2007). It has been noted that attitudinal and social factors play a significant role in an individual's perception of illness, which in turn may affect her chance to take an action such as seeking professional care and subsequently adherence to prescribed medical therapies (Greenwald et al., 1978).

Caretakers sought care for their sick children from various providers. Caretakers' most common source of treatment for febrile illnesses included home management with either traditional practices (Makundi et al., 2006) or stocked antimalarials (Simba \& Kakoko, 2012; Eriksen et al. 2005; Nsimba et al., 2002). Others included biomedical care, mostly from public and private health facilities (Savigny et al., 2004), drug shops, and ADDO (Simba \& Kakoko, 2012). Caretakers' perceptions and belief about the illness influenced the decision of which provider to seek care from (Savigny et al., 2004; Makundi et al., 2006; Warsame et al., 2007; Foster \& Vilendrer, 2009). Public health facilities were the preferable facilities by caretakers of children who experienced an episode of fever. In contrast, caretakers of children with symptoms of convulsions sought care most preferably from traditional healers. Their choice was informed by the belief that convulsion was caused by evil spirits thus, was best cured outside the modern 
health system (Savigny et al., 2004; Makundi et al., 2006; Warsame et al., 2007; Foster \& Vilendrer, 2009). Furthermore, caretakers' subjective assessment of the illness influenced timing of the decision to seek medical care at a health facility. These results are consistent with evidence from studies conducted elsewhere (Mwabu, 1986; Asenso-Okyere et al., 1996).

The proportion of under-five children suspected of malaria who took antimalarial drugs has been observed to decline in recent years. The percentages (both overall and across zones) observed in the 2004-05 survey were generally higher than those in the 2007-08 survey. A slight increase was noted in the 2010 survey however, still below that of the 2004-05. This decline could be due to scale-up of effective malaria prevention measures such as ITNs observed in recent years (Figure 1). Reductions in the impact of malaria as result of large-scale deployment of effective malaria prevention and control measures have observed in several recent studies in Tanzania (Mmbando et al., 2010; Jaenisch et al., 2010; Aregawi et al., 2011).

\section{Conclusion}

In conclusion, across zones in Tanzania, the percentage of households owning at least 1 ITN has increased significantly between 2004 and 2010. Likewise, the proportion of under-five children sleeping under an ITN has increased. The difference between ownership and use of ITNs among under-five is barely visible in recent years. This finding is consistent with a reduction in the proportion of children taking antimalarial drugs in recent years. Nevertheless, some differences exist between zones in terms of both coverage of ownership and use of ITNs.

Effective management of malaria in under-five children requires early diagnosis and prompt action. The health belief model postulates that an individual's willingness to take action in response to a health shock is dependent on three key factors: i) the level of threat posed by the health problem as determined by the individual's perception of severity of an illness and susceptibility to it; ii) the individual's perception of benefit to be derived from engaging in a particular course of action weighed against his or her perception of barriers to performing that particular action, and iii) some type of external or internal trigger or prompt to action (Bush \& Iannotti, 1990). Most of the studies reviewed were designed along these three broad areas hence the evidence. There is a dearth of evidence documenting adherence to prescribed medications, thus more work is required.

\section{Acknowledgements}

The author acknowledge with gratitude comments from three anonymous referees. Financial support from the African Economic Research Consortium through the Bill and Melinda Gates Foundation Attachment Grant, contract Ref. No. PT10R02 is also highly acknowledged.

\section{References}

Akin, J.S, Guilkey, D.K. \& Popkin, B.M. (1981) The demand for child health services in the Philippines. Social Science and Medicine 15C, 249-257. 
Aregawi, M. W., Ali, A.S., Al-mafazy, A., Molteni, F., Katikiti, S., Warsame, M., Njau, R.J.A., Komatsu, R., Korenromp, E., Hosseini, M., Low-Beer, D., Bjorkman, A., D'Alessandro, U., Coosemans, M. \& Otten, M. (2011) Reductions in malaria and anaemia case and death burden at hospitals following scale-up of malaria control in Zanzibar, 1999-2008. Malaria Journal 10, 46.

Asenso-Okyere, W.K., Dzator, J.A. \& Osei-Akoto, I. (1996) The behaviour towards malaria care: A Multinomial Logit Approach Social Indicators Research 39, 167-186.

Barnes, K.I., Chanda, P. \& Barnabas, G.A. (2009) Impact of the large-scale deployment of artemether/lumefantrine on the malaria disease burden in Africa: case studies of South Africa, Zambia and Ethiopia. Malaria Journal 8 (Suppl 1), S8.

Bush, P.J. \& Iannotti, R.J. (1990) A Children's Health Belief Model. Medical Care 28, 69-86.

Comoro, C., Nsimba, S.E., Warsame, M., Tomson, G. (2003) Local understanding, perceptions and reported practices of mothers/guardians and health workers on childhood malaria in a Tanzanian district: implications for malaria control. Acta Tropica 87, 305-313.

Dillip, A., Hetzel, M.W., Gosoniu, D., Kessy, F., Lengeler, C., Mayumana, I., Mshana, C., Mshinda, H., Schulze, A., Makemba, A., Pfeiffer, C., Weiss, M.G. \& Obrist, B. (2009) Socio-cultural factors explaining timely and appropriate use of health facilities for degedege in south-eastern Tanzania. Malaria Journal 8, 144.

Eisele, T.P., Larsen, D. \& Steketee, R.W. (2010) Protective efficacy of interventions for preventing malaria mortality in children in Plasmodium falciparum endemic areas. International Journal of Epidemiology 39, i88-i101.

Eng, J.L.V., Thwing, J., Wolkon, A., Kulkarni, M.A., Manya, A., Erskine, M., Hightower, A. \& Slutsker, L. (2010) Assessing bed net use and non-use after long-lasting insecticidal net distribution: a simple framework to guide programmatic strategies. Malaria Journal 9, 133.

Eriksen, J., Nsimba, S.E.D., Minzi, O.M. S., Sanga, A. J., Petzold, M., Gustafsson, L.L., Warsame, M.Y. \& Tomson, G. (2005) Adoption of the new antimalarial drug policy in Tanzania across-sectional study in the community. Tropical Medicine and International Health 10, 1038-1046.

Foster, D. \& Vilendrer, S. (2009) Two treatments, one disease: childhood malaria management in Tanga, Tanzania. Malaria Journal 8, 240.

Getahun, A., Deribe, K. \& Deribew, A. (2010) Determinants of delay in malaria treatmentseeking behaviour for under-five children in south-west Ethiopia: a case control study. Malaria Journal 9, 320.

Greenwald, H.P., Becker, S.W. \& Nevitt, M.C. (1978) Delay and Noncompliance in Cancer Detection: A behavioral perspective for health planners. The Milbank Memorial Fund Quarterly Health and Society 56.

Hill, Z., Kendall, C., Arthur, P., Kirkwood, B. \& Adjei, E. (2003) Recognizing childhood illnesses and their traditional explanations: exploring options for care-seeking interventions in the context of the IMCI strategy in rural Ghana. Tropical Medicine and International Health 8, 668-676.

Jaenisch, T., Sullivan, D.J., Dutta, A., Deb, S., Ramsan, M., Othman, M.K., Gaczkowski R., Tielsch, J. \& Sazawal, S. (2010) Malaria incidence and prevalence on Pemba Island 
before the onset of the successful control intervention on the Zanzibar archipelago. Malaria Journal 9, 32.

Kachur, S.P., Khatib, R.A., Kaizer, E., Fox, S.S., Abdulla, S.M. \& Bloland, P.B. (2004) Adherence to antimalarial combination therapy with sulfadoxine-pyrimethamine and artesunate in rural Tanzania. American Journal of Tropical Medicine and Hygiene 71, 715-722.

Kamat, V.R. \& Nyato, D.J. (2010) Community response to artemisinin-based combination therapy for childhood malaria: a case study from Dar es Salaam, Tanzania. Malaria Journal 9, 61

Makundi, E.A., Malebo, H.M., Mhame, P., Kitua, A.Y. \& Warsame, M. (2006) Role of traditional healers in the management of severe malaria among children below five years of age: the case of Kilosa and Handeni Districts, Tanzania. Malaria Journal 5, 58.

Maslove, D.M., Mnyusiwalla, A., Mills, E.J., McGowan, J., Attaran, A. \& Wilson, K. (2009) Barriers to the effective treatment and prevention of malaria in Africa: A systematic review of qualitative studies. BMC International Health and Human Rights 9, 26.

Matovu, F., Goodman, C., Wiseman, V. \& Mwengee, W. (2009) How equitable is bed net ownership and utilisation in Tanzania? A practical application of the principles of horizontal and vertical equity. Malaria Journal 8, 109.

Mboera, L.E.G., Kamugisha, M.L., Rumisha, S.F., Kisinza, W.N., Senkoro, K.P. \& Kitua, A.Y. (2008) Malaria and mosquito net utilisation among schoolchildren in villages with or without healthcare facilities at different altitudes in Iringa District, Tanzania. African Health Sciences 8, 114-119.

Mboera, L.E.G., Makundi, E.A. \& Kitua, A.Y. (2007) Uncertainty in malaria control in Tanzania: crossroads and challenges for future interventions. American Journal of Tropical Medicine and Hygiene 77(Suppl 6), 112-118.

Mboera, L.E.G., Kamugisha, M.L., Rumisha, S.F., Msangeni, H. A., Barongo, V., Molteni, F. \& Kitua, A.Y. (2006) The relationship between malaria parasitaemia and availability of healthcare facility in Mpwapwa District, central Tanzania. Tanzania Health Research Bulletin 8, 22-27.

McElroy, B., Wiseman, V., Matovu, F. \& Mwengee, W. (2009) Malaria prevention in northeastern Tanzania: patterns of expenditure and determinants of demand at the household level. Malaria Journal 8, 95.

Mmbando, B.P., Vestergaard, L.S., Kitua, A.Y., Lemnge, M.M., Theander, T.G. \& Lusingu, J.P.A. (2010) A progressive declining in the burden of malaria in north-eastern Tanzania. Malaria Journal 9, 216.

MoHSW (2009) Malaria: Medium Term Strategic Plan 2008-2013. National Malaria Control Programme, Ministry of Health and Social Welfare, United Republic of Tanzania.

Munthali, A.C. (2005) Managing malaria in under-five children in a rural Malawian village. Nordic Journal of African Studies 14, 127-146.

Mwabu, G.M. (1986) Health care decisions at the household level: results of a rural health survey in Kenya. Social Science \& Medicine 22, 315-319.

Nsimba, S.E.D. \& Rimoy, G.H. (2005) Self-medication with chloroquine in a Tanzania: a therapeutic challenge for any treatment policy change in the country. Journal of Clinical Pharmacy and Therapeutics 30, 515-519. 
Nsimba, S.E.D., Massele, A.Y., Eriksen, J., Gustafsson, L.L., Tomson, G. \& Warsame, M. (2002) Case management of malaria in under-fives at primary health care facilities in a Tanzanian district. Tropical Medicine and International Health 7, 201-209.

O'Meara, W.P., Mangeni, J.N., Steketee, R. \& Greenwood, B. (2010) Changes in the burden of malaria in sub-Saharan Africa. Lancet Infectious Disease 10, 545-555.

Oresanya, O.B., Hoshen, M. \& Sofola, O.T. (2008) Utilization of insecticide-treated nets by under-five children in Nigeria: Assessing progress towards the Abuja targets. Malaria Journal 7, 145.

Pokhrel, S., Snow, R., Dong, H., Hidayat, B., Flessa, S. \& Sauerborn, R. (2005) Gender role and child health care utilization in Nepal. Health Policy 74, 100-109.

Pokhrel, S. \& Sauerborn, R. (2004). Household decision-making on child health care in developing countries: the case of Nepal. Health Policy and Planning 19, 218-233.

Rugemalila, J.B., Wanga, C.L. \& Kilama, W. L. (2006) Sixth Africa malaria day in 2006: how far have we come after the Abuja Declaration? Malaria Journal 5,102.

Salah, M.T., Adam, I. \& Malik, E.M. (2007) Care-seeking behaviour for fever in children under five years in an urban area in Eastern Sudan. Journal of Family and Community Medicine $14,25-28$.

Savigny, D. de, Mayombana, C., Mwageni, E., Masanja, H., Minhaj, A., Mkilindi, Y., Mbuya, C., Kasale, H. \& Reid, G. (2004) Care-seeking patterns for fatal malaria in Tanzania. Malaria Journal 3:27.

Simba, D. \& Kakoko, D. (2012). Access to subsidized artemether-lumefantrine from the private sector among febrile children in rural setting in Kilosa, Tanzania. Tanzania Journal of Health Research Doi: http://dx.doi.org/10.4314/thrb.v14i2.1.

Simba, D.O., Kakoko, D.C., Warsame, M., Premji, Z., Gomes, M.F., Tomson, G. \& Johansson, E. (2010) Understanding caretakers' dilemma in deciding whether or not to adhere with referral advice after pre-referral treatment with rectal artesunate. Malaria Journal 9, 123.

Steketee, R.W. \& Campbell, C.C. (2010). Impact of national malaria control scale-up programmes in Africa: magnitude and attribution of effects. Malaria Journal 9, 299.

Suchman, E.A. (1965) Stage of illness and medical care. Journal of Health and Human Behaviour 6, 114-128.

TDHS (2011) Tanzania Demographic and Health Survey 2010. National Bureau of Statistics, Dar es Salaam, Tanzania and ICF Macro.

TDHS (2005) Tanzania Demographic and Health Survey 2004-05. National Bureau of Statistics, Dar es Salaam, Tanzania and ORC Macro.

Teerawichitchainan, B. \& Phillips, J.F. (2008) Ethnic differentials in parental health seeking for childhood illness in Vietnam. Social Science \& Medicine 66, 1118-1130.

THMIS (2008) Tanzania HIVIAIDS and Malaria Indicator Survey 2007-08. Tanzania Commission for AIDS, Zanzibar AIDS Commission, National Bureau of Statistics, Office of the Chief Government Statistician, and Macro International Inc. Dar es Salaam, Tanzania.

TSPA (2007) Tanzania Service Provision Assessment Survey 2006. National Bureau of Statistics, Dar es Salaam, Tanzania and Macro International Inc.

Warsame, M., Kimbute, O., Machinda, Z., Ruddy, P., Melkisedick, M., Peto, T., Ribeiro, I., Kitua, A., Tomson, G. \& Gomes, M. (2007) Recognition, perceptions and treatment practices 
for severe malaria in rural Tanzania: Implications for Accessing Rectal Artesunate as a Pre-Referral. PloS ONE 2(1), e149.

WHO (2011) World Malaria Report. World Health Organisation:

http://www.who.int/malaria/world_malaria_report_2011/9789241564403_eng.pdf.

WHO (2008) World Malaria Report. World Health Organisation:

http://whqlibdoc.who.int/publications/2008/9789241563697_eng.pdf.

Wiseman, V., Scott, A., Conteh, L., McElroy, B. \& Stevens, W. (2008) Determinants of provider choice for malaria treatment: Experiences from The Gambia. Social Science $\mathcal{E}$ Medicine $67,487-496$. 